'gerüst, kanzel', andererseits die bedeutung 'anhöhe, burg'. Diez meint, dass jene sich aus dieser entwickelt habe. Meines erachtens haben wir es mit zwei verschiedenen, unverwanten wörtern zu tun. Pergamo 'burg' ist mit gr. $\pi v$ zubringen. Die versuchung liegt nahe auch prov. perga 'stange' mit unserer sippe zu verknüpfen, aber dieses wort muss fern bleiben. Durch eine form wie pertga bestätigt sich die herleitung aus lat. pertica.

LUND, juli $1907 . \quad$ HERBERT PETERSSON.

\title{
NOCHMALS AS. GENESIS 323.
}

In band $32,567 \mathrm{f}$. dieser zeitschrift habe ich das sinnlose bidoð von as. Genesis 323 in bidrôs oder bidôf zu bessèrn vorgeschlagen. Nun liegt aber bidrồs doch von bidođ graphisch zu weit ab, als dass die conjectur direct überzeugend genannt werden könnte, wenn auch im ae. das part. prt. bedroren und im Heliand einmal das verbum driosan 'hinfallen' vorkommt. Das der handschriftlichen überlieferung näherstehende bidôf oder bidôt (ae.bedeaf) hat den umstand gegen sich, dass ein verbum *dîtan im niederdeutschen nicht vorkommt! Nun bleibt noch eine dritte möglichkeit, an die ich damals nicht gedacht habe, nämlich in bidoð einen schreibfehler für bidôc zu sehen (veranlasst durch das folgende dôठd), wobei dôc das praet. von *dîcan 'tauchen' sein würde. Im as. ist dies allerdings nicht belegt, wol aber die ableitung dîcari 'taucher' (Wadstein, Kl. denkm. s. 178a) und darauf gehen zurück mnd. und nnd. dîken = nnl. duiken. Das mnd. kennt sogar, was meinen neuen besserungsvorschlag auf schönste bestätigt, das compositum be-dûken 'eintauchen'! Ich möchte also v. 323 jetzt lesen: ac sô bidôc it an dôđsêu, sô it noh te daga stendit'.

KIEL, 12. september 1907. F. HOLTHAUSEN. 